\title{
Response to Albuterol MDI Delivered Through an Anti-Static Chamber During Nocturnal Bronchospasm
}

\author{
Sreekala Prabhakaran MD, Jonathan Shuster PhD, \\ Sarah Chesrown MD PhD, and Leslie Hendeles PharmD
}

\begin{abstract}
BACKGROUND: Decreasing electrostatic charge on valved holding chambers increases the amount of drug delivered. However, there are no data demonstrating that this increases bronchodilatation. OBJECTIVE: To investigate the influence of reducing electrostatic charge on the bronchodilator response to albuterol inhaler during nocturnal bronchospasm. METHODS: This randomized double-blind, double-dummy crossover study included subjects, 18-40 years old, with nocturnal bronchospasm (20\% overnight decrease in peak flow on 3 of 7 nights during run-in), $\mathrm{FEV}_{1} \mathbf{6 0 - 8 0 \%}$ predicted during the day, and $\geq 12 \%$ increase after albuterol. Subjects slept in the clinical research center up to 3 nights for each treatment. $\mathrm{FEV}_{1}$ and heart rate were measured upon awakening spontaneously or at 4:00 $\mathrm{AM}$, and $15 \mathrm{~min}$ after each dose of 1, 2, and 4 cumulative puffs of albuterol via metered-dose inhaler. The drug was administered through an anti-static valved holding chamber (AeroChamber Plus Z-Stat) or a conventional valved holding chamber containing a static charge (AeroChamber Plus). RESULTS: Of 88 consented subjects, 11 were randomized and 7 completed the study. Most exclusions were due to lack of objective evidence of nocturnal bronchospasm. Upon awakening, $\mathrm{FEV}_{1}$ was $44 \pm 9 \%$ of predicted before the anti-static chamber and $48 \pm 7 \%$ of predicted before the static chamber. The mean $\pm \mathrm{SD}$ percent increase in $\mathrm{FEV}_{1}$ after 1,2 , and 4 cumulative puffs using the anti-static versus the static chamber, respectively, were $52 \pm 26 \%$ versus $30 \pm 19 \%, 73 \pm 28 \%$ versus $48 \pm 26 \%$, and $90 \pm 34 \%$ versus $64 \pm 35 \%$. The point estimates for the differences (and $95 \%$ CIs) between the devices (anti-static vs static) were $21 \%(4-38 \%)(P=.03), 23 \%(6-41 \%)(P=.02)$, and $25 \%(7-42 \%)(P=.01)$ for 1,2 , and 4 cumulative puffs, respectively. There was no significant difference in heart rate between treatments. CONCLUSIONS: Delivery of albuterol through an anti-static chamber provides a clinically relevant improvement in bronchodilator response during acute, reversible bronchospasm such as nocturnal bronchospasm. Key words: albuterol; anti-static; valved holding chamber; nocturnal bronchospasm. [Respir Care 2012;57(8):1291-1296. ( 2012 Daedalus Enterprises]
\end{abstract}

\section{Introduction}

Efficient delivery of inhaled medications from a metered-dose inhaler (MDI) requires optimal inhalation tech-

Drs Prabhakaran and Chesrown are affiliated with the Pediatric Pulmonary Division, Department of Pediatrics; Dr Shuster is affiliated with the Department of Health Outcomes and Policy; and Dr Hendeles is affiliated with the College of Pharmacy, University of Florida, Gainesville, Florida.

Dr Hendeles presented a version of this paper at the annual meeting of the American Academy of Allergy, Asthma, and Immunology, held March 19, 2011, in San Francisco, California.

The authors have disclosed relationships with Monaghan Medical and Schering-Plough. This research was partly supported by a grant from the Children's Miracle Network at the University of Florida; by fellowship nique. However, many patients have difficulty coordinating actuation and inhalation from an MDI, which impairs drug delivery. ${ }^{1}$ This problem can be solved with the use of a valved holding chamber (VHC) with mouth piece, or

\footnotetext{
training grant T72MC000002 from the Maternal and Child Health Bureau, United States Department of Health and Human Resources; by grant 1UL1RR029890 from the National Center for Research Resources, National Institutes of Health; and by grant RR17568 from the Research Facilities Construction Program, National Institute of Health.

Correspondence: Leslie Hendeles PharmD, College of Pharmacy, University of Florida, 1600 SW Archer Road, Gainesville FL 32610-0486. E-mail: hendeles@cop.ufl.edu.
}

DOI: $10.4187 /$ respcare. 01572 
with an attached mask for children and the elderly. A VHC holds the aerosol in a reservoir prior to inhalation, thereby reducing oropharyngeal deposition and increasing lung deposition. ${ }^{2}$ Larger particles are retained in the VHC, thus allowing smaller particles to be inhaled. ${ }^{3}$

The electrostatic charge (ESC) on the inner walls of conventional chambers attracts aerosol particles and markedly reduces the respirable dose. ${ }^{4}$ This, in turn, reduces the amount of drug delivered to the airways. In contrast, VHCs made from electrically conductive materials emit a significantly larger respirable dose than those made from nonconducting materials, even with wash/rinse pretreatment. ${ }^{5}$

An early in vivo study in children, using plasma concentrations as an indirect measure of the amount of albuterol delivered to the airways by MDI containing a hydrofluoroalkane (HFA) propellant, demonstrated that reducing the ESC on plastic chambers increased lung dose by more than 2-fold. ${ }^{6}$

In contrast, Dompling et al were unable to demonstrate a significant improvement in peak expiratory flow from albuterol MDI containing a chlorofluorocarbon (CFC) propellant, after reducing ESC in VHC with an ionic detergent. ${ }^{7}$ However, the mean peak expiratory flow in these subjects was $91 \%$ predicted, so response was at the upper flat portion of the dose-response curve. Under these circumstances it is not possible to detect a difference in the amount of drug delivered to the airways by measurement of pulmonary function. ${ }^{8}$ In contrast, during nocturnal bronchospasm (drop in $\mathrm{FEV}_{1}$ during the night to $<60 \%$ predicted), the dose-response curve is shifted to the right and much steeper than during the day in the same subjects when asymptomatic. ${ }^{9}$ Consequently, determining bronchodilator response during nocturnal bronchospasm provides a clinically relevant method of comparing differences between delivery devices of the same drug or between different $\beta$ agonists. ${ }^{10}$

There are no data on the clinical relevance of reducing the ESC in patients with acute bronchospasm. Since HFA albuterol may have a greater dose charge than CFC albuterol, ${ }^{11}$ reducing the ESC may have a greater effect than with the former CFC albuterol inhalers. Thus, the purpose of this study was to investigate the influence of reducing ESC on the bronchodilating response to HFA albuterol, using nocturnal asthma as a clinical model of acute bronchospasm. It was our hypothesis that the anti-static chamber would increase bronchodilator response.

\section{Methods}

The study was approved by the University of Florida institutional review board (103-2008), and all subjects gave written informed consent. Eleven subjects, 18-40 years old, with documentation of nocturnal asthma were randomized. None of the randomized subjects were on any

\section{QUICK LOOK}

\section{Current knowledge}

Decreasing the electrostatic charge inside a valvedholding chamber increases the amount of aerosolized drug delivered, but no data demonstrate that this increases the degree of bronchodilatation or dyspnea relief.

\section{What this paper contributes to our knowledge}

Delivery of albuterol through an anti-static chamber, compared to a static chamber, provided a clinically relevant improvement in bronchodilator response during acute, reversible bronchospasm such as nocturnal bronchospasm.

asthma maintenance medications. At the screening visit, subjects had a baseline $\mathrm{FEV}_{1}$ of $60-80 \%$ predicted during the day and a bronchodilator response of $\geq 12 \%$ and to at least $80 \%$ predicted after 2-4 puffs of albuterol HFA MDI delivered by anti-static VHC (Table 1). They had to be nonsmokers for at least 1 year and have a smoking history of not more than 10 pack-years. Women of childbearing age had a negative pregnancy test and used a reliable method of contraception, if sexually active. They were able to perform acceptable and reproducible spirometry according to American Thoracic Society/European Respiratory Society standards for lung function testing. ${ }^{12}$ Subjects were excluded if they had a severe asthma exacerbation requiring hospitalization in the past 12 months, if they required a short course of systemic corticosteroids in the past 30 days, or if they had a viral respiratory infection in the past 3 weeks or during the study.

\section{Study Design}

This was a randomized, double-blind, double dummy, single center, crossover study comparing the bronchodilator response to HFA albuterol MDI delivered through antistatic and static chambers during nocturnal bronchospasm. The first visit was the screening visit. A physical exam, complete blood count, basic metabolic panel, and urinalysis were performed. Then the subjects entered a 7-day run in period where they measured peak expiratory flow twice daily and recorded asthma symptoms and albuterol use in an asthma diary. In order to qualify for the study they had to have a $20 \%$ overnight drop in the peak expiratory flow on 3 out of 7 nights.

After meeting the selection criteria, the subjects slept in the clinical research center up to 3 nights for each treatment, until they woke up spontaneously or had bronchospasm when awakened at 4:00 AM. Baseline $\mathrm{FEV}_{1}$ and vital 


\section{Response to Albuterol}

Table 1. Demographics and Bronchodilator Response During Screening Visit of Subjects Who Completed the Study

\begin{tabular}{|c|c|c|c|c|c|}
\hline $\begin{array}{l}\text { Subject } \\
\text { Number }\end{array}$ & Age, $y$ & Sex & $\begin{array}{l}\text { Baseline } \\
\mathrm{FEV}_{1}, \mathrm{~L}\end{array}$ & $\begin{array}{l}\text { Baseline } \\
\text { FEV }_{1} \text {, } \\
\% \text { predicted }\end{array}$ & $\begin{array}{c}\text { Bronchodilator } \\
\text { Response, } \\
\% \text { increase* }\end{array}$ \\
\hline 18 & 40 & $\mathrm{~F}$ & 1.88 & 63 & 41 \\
\hline 20 & 25 & $\mathrm{~F}$ & 2.04 & 76 & 29 \\
\hline 35 & 18 & $\mathrm{~F}$ & 2.37 & 73 & 19 \\
\hline 36 & 27 & M & 2.72 & 65 & 39 \\
\hline 37 & 21 & M & 3.20 & 62 & 28 \\
\hline 56 & 21 & M & 3.86 & 79 & 19 \\
\hline 87 & 22 & $\mathrm{~F}$ & 2.16 & 75 & 21 \\
\hline Mean \pm SD & $25 \pm 7$ & & $2.60 \pm 0.71$ & $70 \pm 7$ & $28 \pm 9$ \\
\hline
\end{tabular}

signs were performed on admission before 10:00 PM. If subjects did not wake up spontaneously before 4:00 AM, they were awoken by the clinical research center staff at 4:00 AM. Upon awakening, spirometry and heart rate were measured, and the study proceeded if the $\mathrm{FEV}_{1}$ was $\leq 60 \%$ predicted (ie, nocturnal bronchospasm). Subjects then inhaled one actuation from an MDI containing albuterol attached to one of the VHCs, followed by another actuation from an MDI containing placebo attached to the other VHC. This process was repeated with 1 and 2 actuations with both chambers, at 20 min intervals, providing cumulative doses of 1,2, and 4 actuations. There was no delay between releasing the drug dose into the VHC and inhaling. Spirometry and heart rate were repeated 15 min after the last actuation of each dose. The second treatment was identical to the first night, except albuterol was delivered through the opposite active VHC and placebo through the other VHC. Subjects were studied only once on each treatment.

\section{Study Drug}

The drug product used was Proventil MDI, $90 \mu \mathrm{g} / \mathrm{puff}$, while the placebo contained HFA propellant alone, both manufactured by Schering-Plough, Kenilworth, New Jersey. The labels on each canister were covered with an opaque white gummed label. MDIs were primed with several puffs before administration to the subject.

\section{Devices}

AeroChamber Z-Stat Plus was used as the anti-static chamber, and AeroChamber-Plus as the conventional static chamber (both marketed in the United States by Monaghan Medical, Plattsburgh, New York). Each subject used a different set of chambers for each treatment. Blinding of MDI and VHC was performed by an investigator not in- volved in data collection during clinical research center visits (LH).

\section{Electrostatic Charge}

ESC was measured, in duplicate, for each chamber, after removal from the plastic wrapper, with an electrostatic charge meter with a Faraday bucket (NanoCoulomb Meter 284, Monroe Electronics, Lyndonville, New York), which is a battery powered, portable instrument for direct measurement of ESC. The instrument was zeroed and then the chamber was placed in the Faraday bucket and the reading recorded. The anti-static device used for each subject had to have $<2.5$ volts, while the static device had to have $>12$ volts. Interestingly, several static chambers did not have a sufficient ESC and were discarded.

\section{Spirometry}

Spirometry was performed with a calibrated pneumotachometer spirometer (KoKo, Quantum Research, Louisville, Colorado). All $\mathrm{FEV}_{1}$ measurements were performed in duplicate. A third measurement was not planned, since we expected subjects to have moderate to severe airway obstruction and to be in acute distress, requiring albuterol for relief. The clinical research center nurses were trained to perform spirometry. The software was set to utilize the reference equations for predicted values from Hankinson et al. ${ }^{13}$

\section{Statistical Methods}

Sample Size Calculation. The study was designed to have at least $80 \%$ power at $P=.0167(0.05 / 3$ puff variables) two-sided, to detect a difference of $9.4 \%$ in FEV percentages, based on a sample size of 5 per ordering. The actual sensitivity based on a retrospective power calcula- 
tion for $n=7$, was about a $12 \%$ difference. There was no bias, since the decision to terminate the study after 7 completed subjects was made while the data were blinded. This decision was based on the inability to recruit more subjects during the time available to the pulmonary fellow to complete the study.

Randomization. SAS Proc Plan was used to randomize the subjects, and a pharmacist co-investigator (LH) blinded the inhalers and dispensed medication to the clinical research center.

Data Analysis. The primary variable for bronchodilator response was the $\mathrm{FEV}_{1}$. Values for measurements obtained after dosing were expressed as a percent increase from $\mathrm{FEV}_{1}$ upon awakening as follows:

$\left[\left(\right.\right.$ Post-dose $\mathrm{FEV}_{1}-\mathrm{FEV}_{1}$ upon awakening $\left.) \times 100\right] /$

$\mathrm{FEV}_{1}$ upon awakening

The 2-sample method for inference in crossover studies was used, where the period 2 minus period 1 outcomes were obtained independent of treatment ordering, and the orderings compared. Compared to one sample methods that ignore treatment order, this is more robust against bias when the number of subjects assigned to the 2 treatments differs, or where there is a period effect. A univariate analyses was performed on percentage increase in $\mathrm{FEV}_{1}$ at 1, 2, and 4 puffs, while the overall 8-sided bronchodilator response was determined by the Hotelling $\mathrm{T}^{2}$ multivariate analysis. ${ }^{14}$ Heart rate was compared in a similar manner.

\section{Results}

A total of 88 subjects signed the informed consent, but only 11 subjects were randomized (Fig. 1). Most exclusions were due to lack of objective evidence of nocturnal bronchospasm either during the screening visit or during the run-in. Seven subjects completed the study (Table 2). Four subjects did not have nocturnal bronchospasm in the clinical research center. All 7 subjects who completed the study were taking only a short-acting bronchodilator as needed prior to the study.

Upon admission to the clinical research center before $10 \mathrm{PM}$, the evening $\mathrm{FEV}_{1}$ was $62.7 \pm 15 \%$ predicted on the night that they received active drug through the anti-static chamber, and $64 \pm 8.6 \%$ when they received albuterol through the static chamber. Upon awakening, the FEV was precipitously lower than the admission values: $44 \pm 9 \%$ predicted before delivery of albuterol through the antistatic chamber, and $48 \pm 7 \%$ predicted before delivery of active drug through the static chamber.

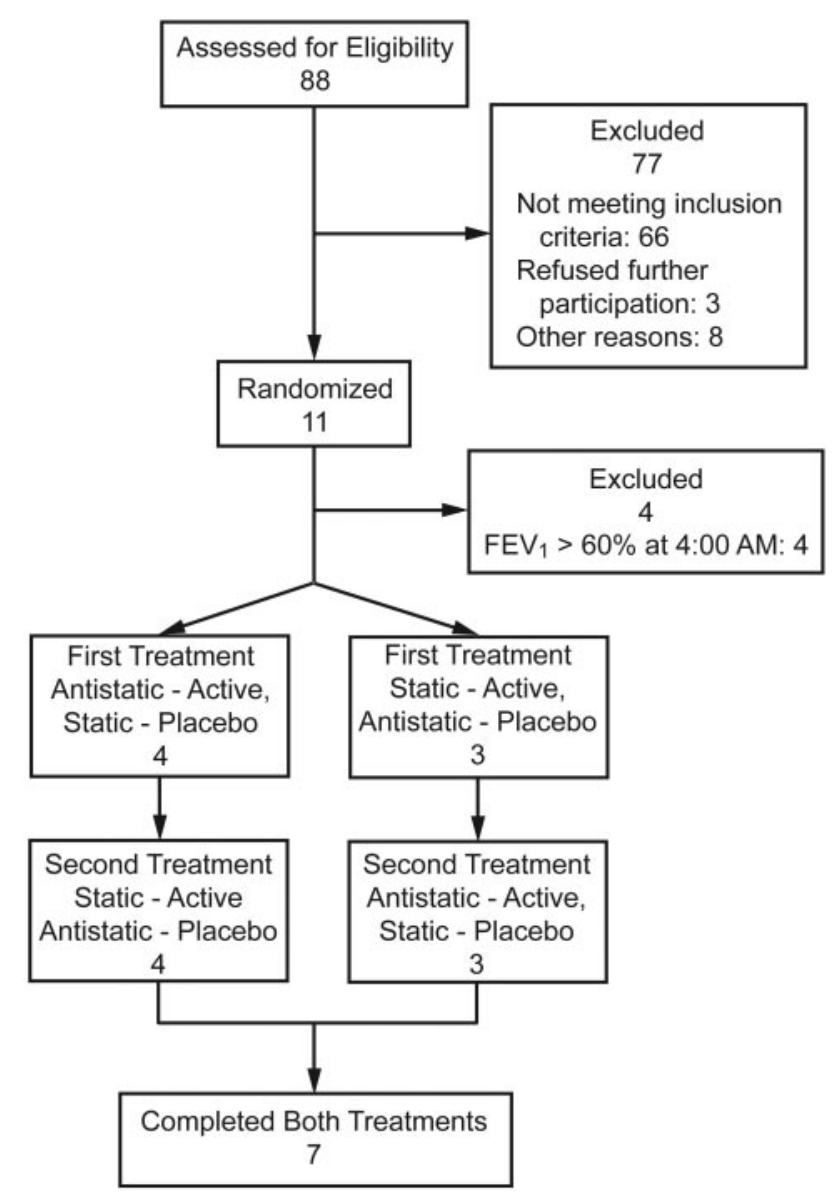

Fig. 1. Consort diagram of subject disposition.

The mean \pm SD percent increase in $\mathrm{FEV}_{1}$ after 1,2 , and 4 cumulative puffs using anti-static versus static chambers, respectively, were $52 \pm 26 \%$ versus $30 \pm 19 \%, 73 \pm 28 \%$ versus $48 \pm 26 \%$ and $90 \pm 34 \%$ versus $64 \pm 35 \%$ (Fig. 2, Table 2). The point estimates (and 95\% CIs) for the difference between treatments (antistatic-static) were $21 \%$ $(4-38)(P=.03), 23 \%(6-41)(P=.02)$, and $25 \%(7-42)$ $(P=.01)$, respectively. However, the overall $\mathrm{T}^{2}$ was not significant $(P=.20)$, possibly due to its 8 -sided nature or to high correlations among the 3 end points. There was no significant carryover effect between treatments.

There were no significant differences in heart rate between the 2 treatment nights (Fig. 3). Also, there were no adverse experiences reported.

\section{Discussion}

The results of this study indicate that the static charge on a VHC decreases bronchodilator response and that eliminating the charge increases bronchodilatation during acute reversible bronchospasm without significantly increasing heart rate. This is the first study, to our knowledge, dem- 
Table 2. Individual Subject Results

\begin{tabular}{|c|c|c|c|c|c|c|c|c|}
\hline $\begin{array}{l}\text { Subject } \\
\text { Number }\end{array}$ & $\begin{array}{c}\text { Baseline } \\
\mathrm{FEV}_{1}^{*}\end{array}$ & $\begin{array}{l}\mathrm{FEV}_{1} \text { at } \\
4: 00 \mathrm{AM}\end{array}$ & $\begin{array}{l}\text { FEV }_{1} \\
\text { After } \\
1 \text { Puff }\end{array}$ & $\begin{array}{c}\% \\
\text { Increase }\end{array}$ & $\begin{array}{c}\text { FEV }_{1} \\
\text { After } \\
2 \text { Puffs }\end{array}$ & $\begin{array}{c}\% \\
\text { Increase }\end{array}$ & $\begin{array}{c}\text { FEV }_{1} \\
\text { After } \\
\text { 4 Puffs }\end{array}$ & $\begin{array}{c}\% \\
\text { Increase }\end{array}$ \\
\hline \multicolumn{9}{|c|}{ Antistatic Chamber } \\
\hline 18 & 2.19 & 1.74 & 2.24 & 29 & 2.47 & 42 & 2.71 & 56 \\
\hline 20 & 1.91 & 1.07 & 2.00 & 87 & 2.05 & 92 & 2.25 & 110 \\
\hline 35 & 1.17 & 1.04 & 1.42 & 36 & 1.80 & 73 & 2.08 & 100 \\
\hline 36 & 2.73 & 1.60 & 3.00 & 88 & 3.55 & 122 & 3.99 & 149 \\
\hline 37 & 3.40 & 2.17 & 3.10 & 43 & 3.42 & 58 & 3.79 & 75 \\
\hline 56 & 4.13 & 2.86 & 3.62 & 27 & 4.19 & 47 & 4.39 & 53 \\
\hline 87 & 1.69 & 1.43 & 2.21 & 54 & 2.52 & 76 & 2.64 & 85 \\
\hline Mean $\pm \mathrm{SD}$ & $2.46 \pm 1.03$ & $1.70 \pm 0.64$ & $2.50 \pm 0.76$ & $52 \pm 26$ & $2.90 \pm 0.88$ & $73 \pm 28$ & $3.10 \pm 0.90$ & $90 \pm 34$ \\
\hline \multicolumn{9}{|c|}{ Static Chamber } \\
\hline 18 & 2.24 & 1.70 & 2.15 & 26 & 2.42 & 42 & 2.50 & 47 \\
\hline 20 & 1.38 & 1.20 & 1.67 & 39 & 1.85 & 54 & 2.03 & 69 \\
\hline 35 & 1.99 & 1.45 & 1.73 & 19 & 1.91 & 32 & 2.37 & 63 \\
\hline 36 & 2.38 & 1.47 & 2.42 & 70 & 2.98 & 110 & 3.51 & 147 \\
\hline 37 & 3.64 & 2.72 & 3.17 & 17 & 3.55 & 31 & 3.85 & 42 \\
\hline 56 & 3.85 & 2.68 & 3.31 & 24 & 3.86 & 44 & 4.02 & 50 \\
\hline 87 & 2.04 & 1.88 & 2.18 & 16 & 2.45 & 30 & 2.60 & 38 \\
\hline Mean $\pm S D$ & $2.50 \pm 0.90$ & $1.87 \pm 0.60$ & $2.40 \pm 0.60$ & $30 \pm 0.2$ & $2.70 \pm 0.78$ & $49 \pm 30$ & $3.00 \pm 0.80$ & $65 \pm 38$ \\
\hline
\end{tabular}

* On admission to the clinical research center in the evening.

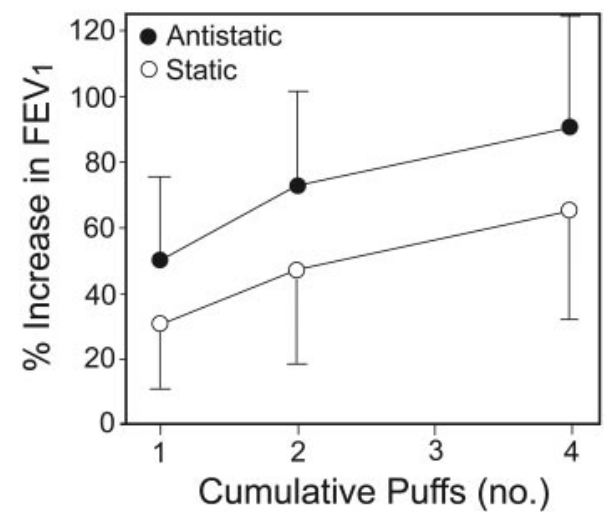

Fig. 2. The mean bronchodilator response expressed as percent increase in $\mathrm{FEV}_{1} 15$ min after 1, 2, and 4 cumulative puffs of albuterol delivered by anti-static chamber or static chamber, in a crossover design, on different nights during nocturnal bronchospasm. There was a significant difference between treatments at each dose. The error bars represent standard deviations.

onstrating a clinically relevant benefit from delivering a short-acting $\beta_{2}$ agonist through an anti-static chamber. It is likely that we were able to measure a difference between devices because the subjects had severe reversible airway obstruction upon awakening. This placed them on the ascending linear portion of the dose-response curve, where differences in dose delivered to the airways results in differences in improvement in $\mathrm{FEV}_{1}$.

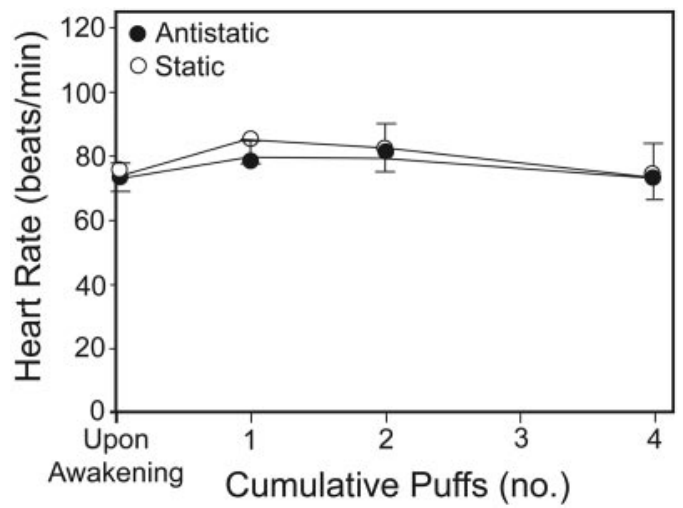

Fig. 3. Heart rate upon awakening with nocturnal bronchospasm and 15 min after 1,2, and 4 cumulative puffs of albuterol delivered by anti-static or static chamber, in a crossover manner, on different nights. There was no significant difference in heart rate between the 2 treatments, because of the low cumulative dose. The error bars represent standard deviations.

In previous studies of bronchodilator response during nocturnal bronchospasm, heart rate increased in a dosedependent manner.9,10 However, 14-16 cumulative puffs were administered in those studies, whereas only 4 cumulative puffs were administered in the present study. Thus, while the anti-static VHC increased the amount of albuterol delivered to the airways, the amount of systemically absorbed drug was probably too low to produce a measurable effect on heart rate. 


\section{Response to Albuterol}

The main limitation of this study is the small sample size. However, that was not because of lack of effort. We screened 88 subjects but could only complete 7 . This indicates the need for multiple centers when using nocturnal bronchospasm as a clinical model. In fact, we had considered using a bioassay by methacholine challenge ${ }^{15}$ to determine whether a difference in dose delivered could be detected, but chose the nocturnal model since it is more clinically relevant.

The results of this study support prescribing an antistatic VHC to deliver albuterol during an episode of acute bronchospasm. For patients who already have a conventional VHC, ESC can be markedly reduced by washing the device with an ionic detergent, and instead of rinsing, allowing the chamber to air dry. ${ }^{16}$

Previous studies in the emergency department treatment of acute asthma indicate that delivering albuterol by MDI through a VHC is at least as effective as delivery by nebulizer but faster, more convenient, and cheaper. ${ }^{17}$ Also, in children, the MDI+VHC method is associated with less tachycardia than the nebulizer method. ${ }^{18}$ Therefore, it would be worthwhile comparing the 2 types of VHCs in an emergency department study.

\section{Conclusions}

In conclusion, delivery of albuterol through an antistatic chamber provides a clinically relevant improvement in bronchodilator response during acute, reversible bronchospasm such as nocturnal bronchospasm.

\section{REFERENCES}

1. Crompton, GK. Problems patients have using pressurized aerosol inhalers. Eur J Respir Dis 1982;119(Suppl):101-104.

2. Newman SP, Newhouse MT. Effect of add-on devices for aerosol drug delivery: deposition studies and clinical aspects. J Aerosol Med 1996;9(1):55-70.

3. Dolovich MA, MacIntyre NR, Anderson PJ, Camargo CA Jr, Chew $\mathrm{N}$, Cole $\mathrm{CH}$, et al; American Association for Respiratory Care. Consensus statement: aerosols and delivery devices. Respir Care 2000; 45(6):589-596.
4. Wildhaber JH, Devadason SG, Hayden MJ, James R, Dufty AP, Fox RA, et al. Electrostatic charge on a plastic spacer device influences the delivery of salbutamol. Eur Respir J 1996;9(9):1943-1946.

5. Rau JL, Coppolo DP, Nagel MW, Avvakoumova VI, Doyle CC, Wiersema KJ, Mitchell JP. The importance of nonelectrostatic materials in holding chambers for delivery of hydrofluoroalkane albuterol. Respir Care 2006;51(5):503-510.

6. Anhøj J, Bisgaard H, Lipworth BJ. Effect of electrostatic charge in plastic spacers on the lung delivery of HFA-salbutamol in children. Br J Clin Pharmacol 1999;47(3):333-336.

7. Dompeling E, Oudesluys-Murphy AM, Janssens HM, Hop W, Brinkman JG, Sukhai RN, de Jongste JC. Randomised controlled study of clinical efficacy of spacer therapy in asthma with regard to electrostatic charge. Arch Dis Child 2001;84(2):178-182.

8. Ahrens RC. On comparing inhaled beta adrenergic agonists. Ann Allergy 1991;67(3):296-298

9. Hendeles L, Beaty R, Ahrens R, Stevens G, Harman EM. Response to inhaled albuterol during nocturnal asthma. J Allergy Clin Immunol 2004;113(6):1058-1062.

10. Hendeles L, Marshik PL, Ahrens R, Kifle Y, Shuster J. Response to nonprescription epinephrine inhaler during nocturnal asthma. Ann Allergy Asthma Immunol 2005;95(6):530-534.

11. Peart J, Kulphaisal P, Orban JC. Relevance of electrostatics in respiratory drug delivery. Pharmagenerics 2003:84-87. http://www. touchbriefings.com/download.cfm?fileID=285. Accessed May 22, 2012.

12. Miller MR, Hankinson J, Brusasco V, Bargos F, Casaburi R, Coates A, et al. Standardisation of spirometry. Eur Respir J 2005;26(2):319338.

13. Hankinson JL, Odencrantz JR, Fedan KB. Spirometric reference values from a sample of the general US population. Am J Respir Crit Care Med 1999;159(1):179-187.

14. Morrison DF. Multivariate statistical methods. New York: McGrawHill; 1967:148-152.

15. Ahrens RC, Hendeles L, Clarke WR, Dockhorn RJ, Hill MR, Vaughan LM, et al. Therapeutic equivalence of Spiros dry powder inhaler and Ventolin metered dose inhaler. A bioassay using methacholine. Am J Respir Crit Care Med 1999;160(4):1238-1243.

16. Piérart F, Wildhaber JH, Vrancken I, Devadason SG, Le Souëf PN. Washing plastic spacers in household detergent reduces electrostatic charge and greatly improves delivery. Eur Respir J 1999;13(3):673678.

17. Hendeles L, Hatton RC, Coons TJ, Carlson L. Automatic replacement of albuterol nebulizer therapy by metered-dose inhaler and valved holding chamber. Am J Health Syst Pharm 2005;62(10): 1053-1061.

18. Schuh S, Johnson DW, Stephens D, Callahan S, Winders P, Canny GJ. Comparison of albuterol delivered by a metered dose inhaler with spacer versus a nebulizer in children with mild acute asthma. J Pediatr 1999;135(1):22-27. 